\title{
Marcel Prawy und das erste Broadway-Musical im Österreich der Nachkriegszeit
}

RENATE KOCH, GRAZ

\section{Kiss Me, Kate auf zwei Bühnen am selben Abend}

„Das Werk ist der Schöpfungsakt des Genies - und nur, wenn Sie das Werk wirklich verstehen und kennen, wenn Sie alles über die Autoren wissen, über ihre Zeit, nur dann werden Sie auch an der Wiedergabe die wahre Freude erleben. ${ }^{\prime 1}$ Marcel Prawy ${ }^{2}$ wurde in Wien geboren, war polnischer Herkunft und hatte jüdische Wurzeln. Er feierte noch seinen 91. Geburtstag und verstarb acht Wochen später in seiner Heimatstadt Wien. Prawy absolvierte das Universitätsstudium der Rechtswissenschaften und eine Klavierausbildung bei Egon Wellesz ${ }^{3}$. Seine Passion war der beinahe tägliche Besuch der Wiener Staatsoper auf Platz Nummer zwei im Stehparterre links. ${ }^{4}$ In dieser Zeit kam es zu zahlreichen Begegnungen mit Opern-Stars, darunter befand sich auch das Ehepaar Kiepura Eggerth. Jan Wiktor Kiepura ${ }^{5}$ zählte damals - neben Richard Tauber und Josef Schmidt - zu den bekanntesten wie populärsten Tenören. Seine Frau Martha Eggerth ${ }^{6}$, ebenfalls Sängerin, engagierte den redegewandten Marcel Prawy als Privatsekretär. Jan Kiepura gab 1938 sein Debüt an der New Yorker Metropolitan Opera als Rodolfo in Giacomo Puccinis La Bohème und wurde dabei von seiner Frau und Marcel Prawy begleitet. Durch dieses Engagement verhalf Kiepura seinem Privatsekretär zur rettenden Ausreise. Nach einigen Jahren sank die Popularität des Ehepaares und im April 1943 kündigten sie Marcel Prawy mit der Begründung, er habe beider künstlerischen und finanziellen Standard abgesenkt. ${ }^{7}$ Nach dem Ende des Dienstverhältnisses und dem Bruch der Freundschaft trat Prawy im Juni 1943 in die US-Army ein. Er schloss sich einer geheimen Einheit an, deren Absolventen ,Ritchie-Boys'8 genannt

1 ORF Videoservice, 100 Jahre Marcel Prawy. Die wahre Freude erleben, DVD, Film von Heidelinde Rudy und Christoph Wagner-Trenkwitz, Sendedatum 27. Dezember 2001, Wien 2001, [00:00-00:13 Min.].

2 1911-2003, Dramaturg und, Opernführer der Nation' mit mehr als 240 Sendungen im Fernsehen.

3 1885-1974, Professor für Musikwissenschaft.

4 Nachzuhören: ORF Videoservice, Showfieber. Wie das Musical Wien eroberte, DVD, Film von Otto Schwarz, Sendedatum 12. März 2006, Wien 2006, [4:45- 5:30].

5 1902-1966, polnischer Opernsänger.

6 1912-2013, österreichisch-ungarische Operettensängerin und Filmschauspielerin.

7 Vgl. Otto Schwarz, Marcel Prawy ,Ich habe die Ewigkeit noch erlebt'. Ein großes Leben neu erzählt, Wien 2006, S. 94-96.

8 Christian Bauer und Rebekka Göpfert, Die Ritchie Boys. Deutsche Emigranten beim USGeheimdienst, Hamburg 2005. 
wurden. Mehr als 15.000 Männer, darunter ca. 3.000 Deutsche und Österreicher, wurden im Camp Ritchie in Maryland ausgebildet. Sie durchliefen während des Zweiten Weltkriegs ein rigoroses Trainingsprogramm, um auf den Einsatz bzw. die Rückkehr nach Europa vorbereitet zu werden. Trainiert wurden nachrichtendienstliche Tätigkeiten wie das Erkunden der militärischen Pläne des Gegners und eine - für damalige Verhältnisse - moderne psychologische Kriegsführung. In diesem Militärlager wurde mehr diskutiert und weniger kommandiert. Die Amerikaner wollten das Wissen und vor allem die Sprachkenntnisse, frei von Akzent und Dialekt, der Emigranten nutzen, um den Gegner zu verunsichern und ihn zur Kapitulation zu bringen. Marcel Prawy kam durch die Invasion in Frankreich im Juni 1944 wieder nach Europa. Als ausgebildeter Elitesoldat kehrte er nach dem Krieg zurück nach Wien. Ab 1952 engagierte er sich speziell dafür, der Bevölkerung die amerikanische Geschichte und die amerikanische Musik näher zu bringen. Das tat er im Kosmos-Theater ${ }^{9}$ und mit der Wanderbühne. ${ }^{10}$ Aus dem einmalig angedachten Musical-Abend im Kosmos-Theater wurden bis 1955 pro Woche zwei bis drei Musical-Shows mit großem Erfolg. Das U.S.-Wandertheater spielte in zwei- und dreimonatigen Tourneen bis zum Ende der Besatzungszeit in vielen, auch kleinen, österreichischen Orten vor interessiertem Publikum. ${ }^{11}$ Im Jahr 1955 erfolgte die Berufung als Dramaturg an die Wiener Volksoper. Mit seinen Produktionen ${ }^{12}$ verhalf er dem US-amerikanischen BroadwayMusical in Österreich zum Durchbruch.

Die Wiener Volksoper wurde 1898 als Kaiser Jubiläums-Stadttheater eröffnet. Sie war ein Dreispartenhaus bis Prawy den Einfall hatte, eine vierte Sparte - das Musical - zu etablieren. Diese Idee forcierten nach und nach drei Pioniere: der zuständige Leiter der Österreichischen Bundestheaterverwaltung Ernst Marboe, Volksopern-Direktor Franz Salmhofer sowie Prawy selbst. Salmhofer willigte vorerst weniger aus Überzeugung, sondern mehr aus Zuneigung ein. Er kämpfte im eigenen Haus gegen eine hartnäckige Ablehnung sowie den beharrlichen Widerstand des Volksopern-Publikums. Durch die Print-Medien waren in Wien bereits vor der Umsetzung des Broadway-Musicals auf der Bühne der Volksoper mehr Gegner als Befürworter zu verzeichnen. Die einhellige Aussage war stets: wir haben die Operette und brauchen kein Musical!

Prawys Pioniertätigkeit zahlte sich aus. Bereits während der amerikanischen Exiljahre entstanden neben seiner Zuneigung zum Broadway-Musical auch Kontakte, Bekanntschaften und Freundschaften, die er für Wien nutzten wollte. Obwohl er von Salmhofer für die Wahl des Musicals nur eine einzige Auflage bekam - nicht Kiss Me, Kate - entschied er sich für eben dieses Musical und unterstrich seine Entscheidung mit einem außerordentlichen Besetzungsplan. Es sollte in Wien zum österreichisch-amerikanischen Zusammentreffen kommen. Prawy

9 Eröffnet im Herbst 1950 von den Amerikanern in der Siebensterngasse 42, 1070 Wien.

10 Eingerichtet vom United States Information Service - kurz USIS.

11 Größenordnung pro Tournee: 45 Orte mit 90 Aufführungen.

12 Kiss Me, Kate (Feb. 1956), Wonderful Town (Nov. 1956), Annie, Get Your Gun (1957), Porgy and Bess (1965), West Side Story (1968), Show Boat (1971) und Karussell (1972). 
holte Darsteller aus Übersee und besetzte parallel mit heimischen Publikumslieblingen, auch der Dirigent vereinigte in sich Österreich und Amerika: Julius Rudel war in Wien Hernals geboren und in die USA emigriert. Prawy erhielt die Genehmigung der Direktion sowie der Bundestheaterverwaltung und begann rasch mit der Umsetzung. Seine selbst auferlegte Eile, in der Wiener Volksoper Kiss Me, Kate von Cole Porter ${ }^{13}$ und den Librettisten Spewack ${ }^{14}$ zu produzieren, hatte drei Gründe: Die deutschsprachige Erstaufführung (DEA) dieses Broadway-Musicals sollte Ende 1955 in Frankfurt am Main über die Bühne gehen. ${ }^{15}$ Überdies gab es am Theater an der Wien ebenfalls Überlegungen, ein Musical zu produzieren. ${ }^{16}$ Außerdem wurde an der Grazer Oper die Erstaufführung (EA) des Musicals als ,Revue-Operette' vorbereitet. ${ }^{17}$

Die aus dem 16. Jahrhundert stammende Vorlage The Taming of the Shrew - zu Deutsch Der Widerspenstigen Zähmung - mit einem Vorspiel und fünf Akten stammt von William Shakespeare ${ }^{18}$, der in einer Person Schauspieler, Autor, Theaterbesitzer, Produzent und Regisseur war. Das Theaterstück atmet den Geist der italienischen Commedia dell'Arte. Die Handlung spielt in Padua, wo der reiche Kaufmann und Edelmann Baptista seine beiden Töchter Katharina und Bianca verheiraten will. Die jüngere, Bianca, hätte mehrere Bewerber, zuerst muss aber ihre ältere Schwester unter die Haube kommen. Lucentio, der Sohn des Edelmanns Vincentio aus Pisa, ist in Bianca verliebt, räumt Mitbewerber aus dem Weg und sucht einen passenden Mann für Katharina. Da kommt Petruchio aus Verona auf der Suche nach einer reichen Partie des Weges und stellt sich als ebenbürtiger Gegner für Katharina dar - er nimmt sie gegen ihren Willen zur Frau. Petruchio zähmt sein grobes Kätchen durch Heirat, Schlafentzug und Hunger; ihre Eitelkeit wird gebrochen und ihre Meinung vernichtet. Tatsächlich aber erweckt er ihre Liebe, und diese Liebe zu ihm lässt sie zur gehorsamen Gattin werden. Petruchio ist nur nach außen ein Grobian, Kätchen sieht seine starke Männlichkeit und sein verstecktes Herz - sie wird gebändigt und folgt inm als Gehorsame.

Durch den Komponisten Cole Porter und das Ehepaar Spewack entstand aus dem Theaterstück das Musical Kiss Me, Kate. Die Basis dieser ,amerikanischen Operette' ${ }^{\prime 19}$ liegt im englischen Drama der Renaissance, in der Tradition einer europäischen Kulturepoche des 15. und 16. Jahrhunderts. Das Musical spielt in Amerika Mitte des 20. Jahrhunderts. Inhaltlich teilt sich das Bühnengeschehen in "onstage" und "backstage" - beide Ebenen sind für das Publikum als ,Spiel im Spiel' zu sehen. Auf der Bühne wird die italienische Komödie dargeboten und backstage werden Zerwürfnisse, Ärgernisse sowie Verwechslungen der Protagonisten ausgetragen. „Onstage" herrscht das Lokalkolorit einer italienischen Stadt vor und "backstage" das Umfeld eines

13 1891-1964, US-amerikanischer Komponist.

14 Samuel (1899-1971) verheiratet mit Bella (1899-1990) waren US-amerikanische Librettisten.

15 DEA Kiss Me, Kate war am 19. November 1955 in den Städtischen Bühnen, Kleines Haus im Börsensaal.

16 Umgesetzt wurden die Überlegungen in der Direktionszeit Fritz Klingenbecks zwischen 1962 und 1965.

17 Premieren-Termin für Kiss Me, Kate war geplant im ersten Quartal 1956.

18 1563/64-1616, Dramatiker und Lyriker aus der Grafschaft Warwickshire.

19 Alternative Bezeichnung des Genres ,Musical'. 
amerikanischen Provinztheaters. Auf diese Idee kam Arnold Saint-Subber ${ }^{20}$ nach einem tatsächlichen Erlebnis. Er beobachtete 1935 bei einer Theateraufführung von Der Widerspenstigen Zähmung die beiden Protagonisten, wie sie sich hinter den Kulissen ebenso stritten wie vor dem Publikum. Dieses Geschehen erzählte er Bella Spewack, die begeistert war und die Arbeit an der Geschichte aufnahm. Der umfassende Inhalt sah mehrere Doppelrollen vor: Regisseur Fred Graham übernimmt selbst die Partie des Edelmanns Petruchio und seine Ex-Frau Lilli Vanessi spielt die widerspenstige, kratzbürstige Katharina. Beide streiten sich vor und hinter der Bühne - ganz in Shakespeare Manier. Neben den Hauptdarstellern Graham und Petruchio sowie Vanessi und Katharina wird ein zweites Paar, Lois Lane ${ }^{21}$ alias Bianca und Bill Calhoun alias Lucentio, für Verwechslungen sorgen. Gemeinsam mit Ehemann Sam wurde dem Komponisten Porter der Einfall unterbreitet, doch dieser lehnte zunächst ab. Erst durch weitere Überzeugungsversuche willigte Porter Weihnachten 1947 ein, sich an der Umsetzung des Musicals zu beteiligen. Nach informellen Gesprächen wurden die Verträge Ende März 1948 unterzeichnet. Nach Tryouts ${ }^{22}$ Anfang Dezember 1948 in New York ${ }^{23}$ und in Philadelphia ging die Uraufführung am 30. Dezember 1948 am NY Broadway im New Century Theatre, bestehend aus zwei Akten, zehn Bildern und sechszehn Szenen, über die Bühne. ${ }^{24}$ Kiss Me, Kate wurde en suite über tausendmal gespielt. Die Europa-Premiere fand am 8. März $1951 \mathrm{im}$ Londoner Coliseum in West End statt und wurde öfter als vierhundertmal gezeigt.

"Cole Porter fügte Satire, Poesie und Sentiment in einer Weise zum Klangkunstwerk wie in keinem seiner Werke davor und danach. ${ }^{25}$ Für die DEA zeichneten Harry Buckwitz ${ }^{26}$ für die Regie, Christoph von Dohnányi ${ }^{27}$ für die musikalische Leitung und Günter Neumann ${ }^{28}$ für die Übersetzung verantwortlich. Kiss Me, Kate blieb ein jahrzehntelanger Erfolg. Die deutsche Bearbeitung textete Marcel Prawy mit entsprechenden österreichischen Worten neu und richtete sie für die Wiener Volksoper ein. Prawy engagierte Julius Rudel ${ }^{29}$, den ersten Dirigenten der New York City Opera Company als musikalischen Leiter. Bei den Protagonisten setzte Prawy auf die angekündigte Mischung von österreichischen Theatergrößen und Darstellern aus Amerika. Der Publikumsliebling Fred Liewehr ${ }^{30}$ spielte die männliche Hauptrolle im Doppelformat

20 1918-1994, US-amerikanischer Produzent.

21 Lois Lane war der Name im Original, später wurde daraus Ann Lane oder Mickey Lane.

22 Oder ,Previews' bezeichnen Testtourneen (on the Road) von zwei bis vier Wochen. Es handelt sich dabei um spezifische Voraufführungen von Broadway-Musicals.

23 Shubert Theatre am 2. Dezember 1948.

24 Thomas Siedhoff, Handbuch des Musicals. Die wichtigsten Titel von A bis Z, Mainz 2007, S. 304.

25 Alexander Bartl, „Küss mich, Kätchen. Kiss Me Kate. USA 1953 f(arbig) 109 min”, in: Filmgenres. Musical- und Tanzfilm, hrsg. von Dorothee Ott und Thomas Koebner, Stuttgart 2014, S. 155.

26 1904-1987, deutscher Regisseur, Intendant, Schauspieler.

$27 * 1929$, deutscher Dirigent und Intendant.

28 1913-1972, deutscher Kabarettist und Texter.

29 1921-2014, in Wien geborener US-amerikanischer Dirigent.

30 1909-1993, österreichischer Schauspieler, Operettensänger, Intendant. 
und an seiner Seite als weibliche Hauptdarstellerin - ebenfalls in der Doppelrolle - glänzte die amerikanische Sängerin Brenda Lewis ${ }^{31}$. Die Melange aus Amerika und Österreich setzte sich fort mit Olive Moorefield ${ }^{32}$ sowie Hubert Dilworth ${ }^{33}$ und Helmut Qualtinger ${ }^{34}$ sowie Kurt Preger ${ }^{35}$. Diesem außergewöhnlichen Erfolg der ÖEA von „Kiss Me, Kate” am 14. Februar 1956 folgten bis 1972 insgesamt 183 Vorstellungen. „Kiss me Kate, diese ERSTE GROSSE EUROPÄISCHE MUSICAL-PRODUKTION im amerikanischen Stil, hat die Theaterlandschaft Europas verändert, was mir damals nicht bewußt war," erinnerte sich Marcel Prawy, "es war die ,blitzeste' Blitzproduktion aller Zeiten." ${ }^{136}$ In nur sechs Wochen kam es zur Premiere. Die Brücke zwischen USA und Österreich verband beide Stile zu einer Volksoper-eigenen Darstellungsform und so entstand das Zusammenspiel aus österreichischer traditioneller, musikalischer Kultur mit amerikanischem ureigenem, unnachahmlichem Schwung. ${ }^{37}$ Zu Beginn war die Ablehnung groß, nach der Premiere sonnten sich auch die Gegner in Prawys Triumph. Die Rezensionen in den Tageszeitungen waren überwiegend positiv und wurden mit Worten wie "da capo, da capo, da capo!"38 - „Triumphaler Einzug des Musicals in die Volksoper" ${ }^{\prime \prime 9}$ - "Küß mich, ja, Käthe! ${ }^{40}$ - „eine treffliche Aufführung und ein durchschlagender Erfolg" 41 - "Beifall für [...] exzellente Propagandisten"42 bejubelt. Viele Kritiker beschrieben - neben eigener Eindrücke - auch die Stimmung des beeindruckten Premieren-Publikums.

Am selben Tag - eine halbe Stunde später - begann die EA im Grazer Opernhaus - 14. Februar 1956, 19:30 Uhr. Die Wiener hoben den Vorhang um 19 Uhr, damit niemand die Bezeichnung ÖEA in der Volksoper anzweifeln konnte. In Graz führte der Intendant André Diehl selbst Regie und am Pult stand Rudolf Bibl. Als musikalischer Leiter arrangierte er Porters Musik anhand eines Klavierauszugs für das Orchester. Diese Instrumentierung wurde durch fehlende Musiker notwendig. Auf dem Besetzungszettel stand ,Deutsch von Günther Neumann', allerdings ließ sich beim Vergleich der Textbücher feststellen, dass in Graz gesprochene wie gesungene Passagen mitunter verändert und teilweise eigens konstruiert wurden. Diese Produktion wurde als Revue-Operette bzw. Operetten-Revue bezeichnet und es fehlten partiell Zwischennummern. Im Opernhaus standen heimische Protagonisten auf der Bühne, die allesamt Operetten-

\footnotetext{
31 1921-2017, amerikanische Sopranistin und Musical-Darstellerin.

32 *1932, amerikanische Allrounderin.

33 1910-1994, afro-amerikanischer Sänger.

34 1928-1986, österreichischer Schauspieler und Schriftsteller.

35 1907-1960, österreichischer Tenor und Bariton.

36 Marcel Prawy, "Marcel Prawy erinnert sich an seine Tätigkeit an der Volksoper”, in: Die Volksoper. Das Wiener Musiktheater, hrsg. von Klaus Bachler et al., Wien 1998, S. 153-165, hier S. 154.

37 Ebd., S. 155.

38 Neuer Kurier (15. Februar 1956), S. 5.

39 "Theater und Kunst - Kritik", in: Die Presse Nr. 2223 (Donnerstag, 16. Februar 1956), S. 4.

40 Arbeiterzeitung Nr. 39 (Donnerstag, 16. Februar 1956), S. 4.

41 Österreichische Neue Tageszeitung Nr. 39 (Donnerstag, 16. Februar Jahrgang 1956), S. 4

42 Kleine Zeitung Nr. 39 (Donnerstag, 16. Februar 1956), S. 9.
} 
sänger und/oder Schauspieler waren. Die Premiere fand ,nur' lebhaftes Interesse ${ }^{43}$ und nach 16 Vorstellungen stand Kiss Me, Kate ab November nicht mehr auf dem Spielplan der neuen Saison 1957/58. Die Hauptdarsteller Peter Minich, Eleonore Bauer, Elfie Körner oder Heinz Peters wurden in den Kritiken zwar lobend erwähnt, das Gesamtbild hinterließ jedoch einen weniger bleibenden Eindruck. Die Überschriften der Rezensionen lauteten: „Kätchens ,großer' Fasching-Dienst ${ }^{44}$-Tag im Opernhaus" 45 - „Der Patient war sehr, sehr tapfer und lächelte noch unter Tränen" 46 - "Kätchen ist sehr widerspenstig"47, und ähnliches. Positive Erwähnungen gab es für Peter Minich, der von Prawy schon im Mai 1956 als Ergänzung für Fred Liewehr nach Wien geholt wurde. Er sei ein wirkliches "Manns-Exemplar, das charmant sein, aber auch zuhauen $\mathrm{kann}^{4}{ }^{48}$. Lobende Worte erhielt auch das widerspenstige Kätchen Eleonore Bauer, sie „zeigt eine Person, die zu zähmen sich lohnt"49 (Kleine Zeitung). Zusammenfassend kann festgehalten werden, dass vieles - wahrscheinlich - zu vieles geändert wurde und unter Umständen dazu führte, dass die Grazer Produktion nicht von Erfolg gekrönt war und nur 16 mal zur Aufführung kam.

Bei den Erstaufführungen von Kiss Me, Kate in den Stadt- und Landestheatern wurde stets auf Marcel Prawys Neutextierung und Einrichtung für die Wiener Volksoper Wert gelegt. Viele Faktoren sind ausschlaggebend für einen Erfolg und Prawy wurde, hinsichtlich neuer Textierung der deutschen Fassung von Günter Neumann, zum Garant für das Linzer Landestheater (EA 7. Februar 1957), das Tiroler Landestheater (EA 9. November 1958), das Klagenfurter Stadttheater (EA 19. Juni 1959) und das Salzburger Landestheater (EA 23. April 1960). Die Oberösterreichischen Nachrichten titelten "Kiss mi Kathi! Scheksbier drah di!" ${ }^{40}$ und das Tagblatt setzte die Überschrift "Kiss me, Kate! Begeisterte Aufnahme des ersten Musicals durch Jung-Linz" ${ }^{51}$. In der Tiroler Tageszeitung stand „Broadwayschlager ,Kiss me, Kate' im Landestheater, Hausgemachtes "Musical' gewann sich das Publikum. Ensemble übertraf die Erwartung "52 und in den Tiroler Nachrichten begann die Rezension mit den Worten: „Tolle Sache - tolle Aufführung - toller Erfolg!" ${ }^{43}$. Die Kleine Zeitung in der Ausgabe für Kärnten und Osttirol schrieb „Ein amerikanisches Musical stellt sich vor" ${ }^{\prime \prime}$. In den Salzburger Nachrichten verfasste der Kritiker "Broadway an der Salzach - Respekt vor unserem, entfesselten' Landestheater! Ein so anspruchsvolles Stück [...] mit einem

43 Vgl. Kleine Zeitung Nr. 39 (Donnerstag, 16. Februar 1956), S. 9.

44 Die Formulierung lässt vermuten, dass ,Dienst' im Sinne von Arbeit verstanden werden sollte.

45 Kleine Zeitung Nr. 39 (Donnerstag, 16. Februar 1956), S. 9.

46 Neuer Kurier (15. Februar 1956), S. 5.

47 Neue Zeit Nr. 39 (Donnerstag, 16. Februar 1956), S. 6.

48 Kleine Zeitung Nr. 39 (Donnerstag, 16. Februar 1956), S. 9.

49 Ebd.

50 Oberösterreichischen Nachrichten 93 (Samstag, 9. Februar 1957), Nr. 34, S. 4.

51 „Kunst und Wissenschaft”, Tagblatt 13 (Freitag, 9. Februar 1957), Nr. 45, S. 6.

52 Tiroler Tageszeitung 14 (Dienstag, 11. November 1958), S. 2.

53 Tiroler Nachrichten Nr. 260 (Dienstag, 11. November 1958), S. 4.

54 Kleine Zeitung (Freitag 21. Juni 1959), S. 17. 
derart wirbelnden Schmiß, mit soviel Witz und artistischem Können hinzulegen, das will schon etwas bedeuten" 55 .

Auf die Frage nach seiner größten Bereicherung meinte Prawy: "Seelisch das größte Erlebnis war ,Kiss me, Kate', weil es das erste war. Auf der Bühne die größten waren ,Porgy and Bess' und ,West Side Story'." ${ }^{56}$ Als Fazit sei bemerkt, dass die ÖEA in Wien tosenden Applaus erntete und die Gegner überstimmt waren. Die Grazer EA wurde zur , halbbitteren Angelegenheit'57. Die Stadt- und Landestheater verzeichneten allesamt Erfolge. Zur Freude der Befürworter setzte sich das Musical als neues Genre im Österreich der Nachkriegszeit durch.

Zitation: Renate Koch, "Marcel Prawy und das erste Broadway-Musical im Österreich der Nachkriegszeit", in: Freie Beiträge zur Jahrestagung der Gesellschaft für Musikforschung 2019, hrsg. von Nina Jaeschke und Rebecca Grotjahn (= Musikwissenschaft: Aktuelle Perspektiven. Bericht über die Jahrestagung der Gesellschaft für Musikforschung 2019 in Paderborn und Detmold, Bd. 1), Detmold 2020, S. 142-149, DOI: 10.25366/2020.57.

55 Salzburger Nachrichten (Montag, 25. April 1960), S. 7.

56 Marcel Prawy, Peter Dusek und Christoph Wagner-Trenkwitz, Marcel Prawy erzählt aus seinem Leben, Wien 1997, S. 130.

57 Harald Kaufmann, in: Neue Zeit Nr. 39 (Donnerstag, 16. Februar 1956), S. 6. 


\section{Abstract}

Marcel Prawy, born in Vienna, graduated in law. In 1936, the couple Kiepura/Eggerth engaged him as private assistant. Two years later Jan Kiepura helped him to emigrate to New York. In 1943, after his employment ended, Prawy joined the US Army. Finally he returned as an elite soldier to Vienna and began his pioneering work for 'Broadway Musicals'. In 1955, he was appointed dramaturge at the 'Wiener Volksoper'. One year later in February, Kiss Me, Kate was performed in two Austrian theatres. The Viennese version was produced by Prawy himself and staged by Heinz Rosen. In Graz André Diehl directed the orchestration by conductor Rudolf Bibl on the basis of a piano score. Prawy relied on a mixture of Austrian theatre luminaries and American actors. In the Volksoper 183 performances took place - Graz had only 16 . The reviews for the Viennese premiere reaffirmed the cheers. The criticism of the Graz production did not receive the same attention as Prawy's production did.

\section{Kurzvita}

Renate Koch promovierte im November 2019 an der Kunstuniversität Graz. Ihr studentischer Werdegang beinhaltet einige Semester Volkskunde und Soziologie sowie nach dem Bachelor of Arts die Abschlüsse der Masterstudiengänge Musikologie (2014) und Angewandte Ethik (2016) an der Grazer Universität für Musik und darstellende Kunst sowie der Karl-Franzens-Universität Graz. 


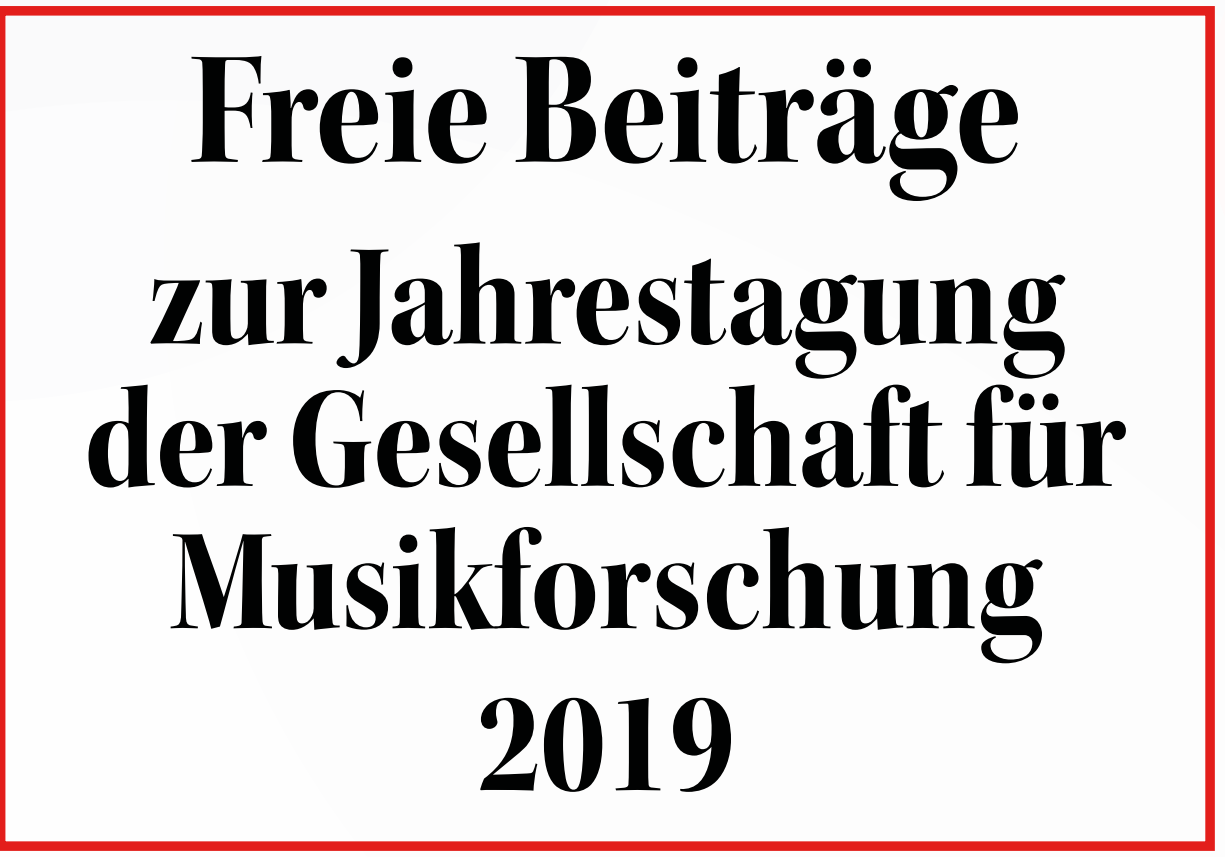

Herausgegeben von Nina Jaeschke und Rebecca Grotjahn

Musikwissenschaft: Aktuelle Perspektiven 1 
Freie Beiträge 


\section{Musikwissenschaft: Aktuelle Perspektiven}

Bericht über die Jahrestagung der Gesellschaft für Musikforschung 2019 in Paderborn und Detmold

Herausgegeben von Rebecca Grotjahn und Nina Jaeschke

Band 1 


\section{Freie Beiträge}

\section{zur Jahrestagung der Gesellschaft für Musikforschung 2019}

Herausgegeben von Nina Jaeschke und Rebecca Grotjahn

Detmold: Musikwissenschaftliches Seminar der Universität Paderborn und der Hochschule für Musik Detmold 2020 
DOI: $10.25366 / 2020.42$

Online-Version verfügbar unter der Lizenz: Urheberrecht 1.0, $<$ https://rightsstatements.org/page/InC/1.0/?language=de>

Bibliografische Information der Deutschen Nationalbibliothek

Die Deutsche Nationalbibliothek verzeichnet diese Publikation in der Deutschen Nationalbibliografie; detaillierte bibliografische Daten sind im Internet über http://dnb.d-nb.de abrufbar.

\section{Impressum}

Redaktion: Nina Jaeschke, Rebecca Grotjahn und Jonas Spieker Satz: Nina Jaeschke

(C) Musikwissenschaftliches Seminar der Universität Paderborn und der Hochschule für Musik Detmold 2020 


\section{INHALT}

Vorwort $\quad$ IX

Komponieren für das Radio: Akteure, Diskurse, Praktiken $\quad 1$

Musikwissenschaft - Feminismus - Kritik: Ein Generationenaustausch 6

\section{Stefan Alschner}

Der Wagner-Sänger Joseph Aloys Tichatschek - Vom Nachlass zum Netzwerk

\section{Alenka Barber-Kersovan}

Songs for the Goddess. Das popmusikalische Neo-Matriarchat zwischen Ethno-Beat,

erfundenen Traditionen und kommerzieller Vermarktung

Elias Berner, Julia Jaklin, Peter Provaznik, Matej Santi, Cornelia Szabó-Knotik

Musikgeschichte anders erzählen? Das Beispiel der 1970er in Österreich.

Musikhistoriographie in der Zeit der Digitalisierung

\section{Mauro Fosco Bertola}

„Ein Laut so klagevoll”. Lohengrin zwischen Richard Wagner und Salvatore Sciarrino

\section{Matthieu Cailliez}

Europäische Rezeption der Berliner Hofoper und Hofkapelle von 1842 bis 1849

\section{lacopo Cividini}

Zwischen klassischer Musikphilologie und angewandter Informatik:

Die Digitale Mozart-Edition (DME) der Stiftung Mozarteum Salzburg

\section{Marko Deisinger}

Fortschrittliche Technologie im Dienste eines Antimodernisten.

Heinrich Schenker und der österreichische Rundfunk

\section{Norbert Dubowy}

Vom Kritischen Bericht zur Kritischen Dokumentation am Beispiel der Digital-interaktiven Mozart-Edition

\section{Markus Engelhardt}

Musik zwischen Nation Building und Internationalität. Italien um 1900

\section{Maryam Haiawi}

Das Oratorium im Spannungsfeld der Konfessionen: 


\section{Judith I. Haug}

"Manch eine*r liegt, morgens noch trunken, im Rosengarten" - Rekonstruktionen

osmanischer Musikgeschichte in Gesangstextsammlungen

\section{Renate Koch}

Marcel Prawy und das erste Broadway-Musical im Österreich der Nachkriegszeit

Susanne Kogler, Julia Mair, Juliane Oberegger, Johanna Trummer

Erich Marckhl - Musikausbildung in der Steiermark nach 1945.

Brüche und Kontinuitäten

\section{Marie-Anne Kohl}

Die weinende Jury. "Geschlechtslose" Tränen bei globalen Musik-Castingshows?

\section{Fabian Kolb}

Tanztheater und filmische Ästhetik. Cineastische Einflüsse und Gestaltungsweisen in den Kompositionen für die Ballets Suédois 1920-1925

\section{Christian Lehmann}

Tempobezeichnungen von Julius Stockhausen für Die schöne Müllerin:

Ein Quellenfund

\section{Martin Link}

Signum et gens - Zur Gendersemiotik in Clara und Robert Schumanns Liederzyklus Liebesfrühling

\section{Livio Marcaletti}

„Strafspiel" und satirische Stilmittel in musikdramatischen Gattungen des frühen 18. Jahrhunderts

\section{Tobias Marx, Martin Lissner}

Thüringer Musikszene - Jugendmusikredaktionen als außerschulische musikbezogene Bildungskontexte

\section{Maho Naito}

Die Parallelität der Entstehungsprozesse der ersten beiden Symphonien Gustav

Mahlers: Instrumentation, Revision und Dirigierpraxis

\section{Elisa Novara}

Eine Schumann-Werkstatt? Zur Übertragbarkeit der Methoden vom Projekt 
Theodora Oancea, Joachim Pollmann, Jonas Spieker

Kollaborateure - Involvierte - Profiteure. Erarbeitung eines Online-Lexikons zur

Musik in der NS-Zeit

\section{Kiron Patka}

„Ich wollte eigentlich Sängerin werden." Berufsselbstbilder von Tontechniker*innen im Radio

\section{Siegwart Reichwald}

Die Leiden der jungen Clara: Das Klaviertrio Opus 17 als Ausdruck einer Neu-

Romantikerin

\section{Elisa Ringendahl}

Lied versus Oper - Pole musikalischer Gattungen bei Oscar Bie

\section{Benedikt Schubert}

Struktur und Exegese. Über Eigentümlichkeiten in der Arie "Des Vaters Stimme ließ sich hören" (BWV 7/4)

Uwe Seifert, Sebastian Klaßmann, Timo Varelmann, Nils Dahmen

Computational Thinking in der Musikwissenschaft: Jupyter Notebook als Umgebung

für Lehre und Forschung

\section{Yusuke Takamatsu}

Synthese als Modus der Prozessualität bei Schubert:

Sein spezifisches Wiederholungsprinzip im langsamen Satz

\section{Daniel Tiemeyer}

Johann Nepomuk Hummels Sonate in fis-Moll Op. 81 - Studien zu Entstehungs-

hintergrund, Rezeption und formalerStruktur

\section{Andrea van der Smissen}

Musikalische Innovation im Umfeld der Moderne und historischen Avantgarde in Ungarn

\section{Tim Ziemer, Holger Schultheis}

Psychoakustische Sonifikation zur Navigation in bildgeführter Chirurgie

\section{Magdalena Zorn}

Musik mit dem Radio hören: Über den Begriff der musikalischen Aufführung 
Gabriele Buschmeier in memoriam 


\section{Vorwort}

Die vorliegenden Bände dokumentieren die Jahrestagung der Gesellschaft für Musikforschung 2019. In den dreieinhalb Tagen vom 23. bis zum 26. September 2019 wurden in Paderborn und Detmold nicht weniger als 185 Beiträge präsentiert, verteilt auf diverse Symposien, Round tables, Freie Sektionen und Postersessions. Sie alle auf einen Nenner bringen zu wollen, ist ein Ding der Unmöglichkeit - und das ist gut so, ist es doch Ziel der Jahrestagungen, die große Vielfalt der Themen und Methoden des Faches Musikwissenschaft abzubilden. Um die thematische Vielfalt der freien Referate angemessen abbilden zu können und gleichzeitig den inhaltlichen Schwerpunkten der beiden hier publizierten Hauptsymposien ausreichend Raum bieten zu können, erscheinen diese in drei Bänden.

„Musikwissenschaft: Aktuelle Perspektiven": Der Titel der kleinen Reihe ist keine Verlegenheitslösung. Musikwissenschaft im Kontext der Digital Humanities; Musikwissenschaft und Feminismus; Musik und Medien; Musikalische Interpretation - schon die Felder, die von den vier Hauptsymposien bespielt wurden, wären noch vor wenigen Jahrzehnten allenfalls an der Peripherie das Faches zu finden gewesen. Sie entsprechen Arbeitsschwerpunkten der Lehrenden am Musikwissenschaftlichen Seminar der Universität Paderborn und der Hochschule für Musik Detmold, das die Tagung ausrichtete. Zugleich nehmen sie Bezug auf aktuelle Ereignisse und Entwicklungen. So erwuchs das von Andreas Münzmay und Joachim Veit organisierte Symposium „Brückenschläge - Informatik und Musikwissenschaft im Dialog" unmittelbar aus den Erfahrungen im Virtuellen Forschungsverbund Edirom (ViFE) und im fakultäten- und hochschulübergreifenden Zentrum Musik-Edition-Medien (ZenMEM). Der 200. Geburtstag von Clara Wieck/Schumann war der Anlass für das von Rebecca Grotjahn geleitete Symposium „Die Begleiterin - Clara Schumann, Lied und Liedinterpretation", das in enger Kooperation mit der Hochschule für Musik Detmold durchgeführt wurde. Das Hauptsymposium „Brückenschläge" wird in einem separaten Band publiziert (Bd. 3 der vorliegenden Reihe). Im Rahmen dieses Symposiums führte die von Stefanie Acquavella-Rauch geleitete Fachgruppe Digitale Musikwissenschaft eine Posterpräsentation durch, die von den Beiträger*innen erfreulicherweise zu kürzeren Texten umgearbeitet wurden, sodass sie hier ebenfalls, zusammen mit den Postern,

publiziert werden können. Hinzu kommen einige Beiträge, die bereits bei der Jahrestagung 2018 in Osnabrück präsentiert wurden. Auch das Hauptsymposium "Die Begleiterin" wird in einem eigenen Band (Bd. 2) publiziert. Die Beiträge zu den beiden anderen Hauptsymposien hingegen werden an anderen Orten veröffentlicht; in Band 1 („Freie Beiträge zur Jahrestagung der Gesellschaft für Musikforschung 2019") der vorliegenden Publikation finden sich jedoch Einführungen und Abstracts. Das Symposium „Komponieren für das Radio" unter Leitung von Antje Tumat und Camilla Bork (Katholieke Universiteit Leuven) behandelte Einflüsse des Mediums auf Kompositionsprozesse sowie durch radiophone Kompositionen bzw. radiophonen Klang ausgelöste Diskurse. Sarah Schauberger und Cornelia Bartsch (Universität Oldenburg) nahmen das 25-jährige Jubiläum der Fachgruppe Frauen- und Genderstudien zum Anlass für einen Generationenaustausch zum Thema "Musikwissenschaft - Feminismus - Kritik": Was wa- 
ren vor einem Vierteljahrhundert Inhalte und Aufgaben einer feministischen Musikwissenschaft und wie kann sich diese heute positionieren?

Bewusst haben wir im Tagungsbericht auf inhaltliche Eingriffe in die Beiträge verzichtet. ${ }^{1}$ Das gilt besonders für die Freien Referate: Es galt, den Charakter der Jahrestagung als Forum für ,freie', d. h. innovative und auch experimentelle Gedanken zu wahren. Einige Kolleg*innen, die die Tagung mit Vorträgen und Posterpräsentationen bereichert hatten, haben sich gegen eine Publikation im vorliegenden Band entschieden - sei es, weil sie eine Möglichkeit fanden, ihre Beiträge in einem inhaltlich passenderen Rahmen zu veröffentlichen, sei es, weil ihre Überlegungen in ihre entstehenden Qualifikationsschriften fließen sollen, oder sei es, weil sie von den Autor*innen in der vorgetragenen Form zunächst verworfen wurden. Auch damit erfüllt eine Freie-Referate-Sektion ihren Zweck: Die Diskussionen mit der versammelten Fach-Öffentlichkeit sollen dabei helfen, Gedanken weiterzuentwickeln und zu verändern. In diesem Sinne sei allen Beteiligten - den Autor*innen, den nichtpublizierenden Referent*innen und den Mit-Diskutant*innen - ganz herzlich gedankt für ihr Mitwirken bei der Tagung.

Unser herzlicher Dank gilt einer Reihe weiterer Personen, die zum Gelingen dieser drei Bände beigetragen haben. Hier ist besonders Jonas Spieker zu nennen, der uns tatkräftig bei der Redaktion geholfen hat. Andrea Hammes (SLUB Dresden) sei herzlich für die Aufnahme unseres Bandes auf musiconn.publish gedankt - wir freuen uns, damit unsererseits zur Etablierung dieser innovativen Publikationsplattform beizutragen.

Erneut möchten wir an dieser Stelle allen Menschen danken, die uns bei der Organisation, Ausrichtung und Finanzierung der Tagung selbst unterstützt haben: der Präsidentin der Universität Paderborn, Prof. Dr. Birgitt Riegraf, dem Rektor der Hochschule für Musik Detmold, Prof. Dr. Thomas Grosse, den Kolleginnen und Kollegen der beiden beteiligten Hochschulen, dem Vorstand der Gesellschaft für Musikforschung, der Universitätsgesellschaft Paderborn und allen Sponsoren. Besonders dankbar sind wir den Mitarbeiter*innen und den studentischen bzw. wissenschaftlichen Hilfskräften des Musikwissenschaftlichen Seminars, die bei der Vorbereitung und Ausrichtung der Tagung immensen Einsatz zeigten - stellvertretend sei an dieser Stelle Johanna Imm erwähnt, die zusammen mit Nina Jaeschke das Herz des Organisationsteams bildete.

Wir widmen diese Reihe Dr. Gabriele Buschmeier, dem langjährigen Vorstandsmitglied der Gesellschaft für Musikforschung, die kurz vor der Publikation dieses Bandes unerwartet verstarb.

Detmold, im September 2020

Rebecca Grotjahn und Nina Jaeschke

Zitation: Rebecca Grotjahn und Nina Jaeschke, „Vorwort”, in: Freie Beiträge zur Jahrestagung der Gesellschaft für Musikforschung 2019, hrsg. von Nina Jaeschke und Rebecca Grotjahn (= Musikwissenschaft: Aktuelle Perspektiven. Bericht über die Jahrestagung der Gesellschaft für Musikforschung 2019 in Paderborn und Detmold, Bd. 1), Detmold 2020, S. IX-X, DOI: 10.25366/2020.43.

1 Freigestellt war den Autor*innen auch, ob sie sich für eine gendersensible Sprache entscheiden bzw. welche Form des Genderns sie bevorzugen. 